\title{
CORPUS Corpus
}

11 | 2012

La cooccurrence, du fait statistique au fait textuel

\section{Nouveau traitement des cooccurrences dans Hyperbase}

Etienne Brunet

\section{(2) OpenEdition \\ 12 Journals}

Édition électronique

URL : http://journals.openedition.org/corpus/2275

DOI : $10.4000 /$ corpus.2275

ISSN : 1765-3126

Éditeur

Bases; corpus et langage - UMR 6039

Édition imprimée

Date de publication : 1 janvier 2012

ISSN : 1638-9808

Référence électronique

Etienne Brunet, « Nouveau traitement des cooccurrences dans Hyperbase », Corpus [En ligne], 11

2012, mis en ligne le 18 juin 2013, consulté le 08 septembre 2020. URL : http://

journals.openedition.org/corpus/2275; DOI : https://doi.org/10.4000/corpus.2275

(C) Tous droits réservés 


\title{
Nouveau traitement des cooccurrences dans Hyperbase
}

\author{
Etienne BRUNET \\ Université Nice Sophia Antipolis, CNRS, BCL, UMR 7320, \\ 06357 Nice, France
}

\section{Cooccurrences simples et thèmes}

1.1 On vise ici à représenter les relations qui lient entre eux les mots d'un texte, ces relations étant considérées dans l'espace étroit du paragraphe et non dans l'espace du texte. Cette fonction, intitulée THEME, est depuis longtemps intégrée à Hyperbase, dans une approche limitée à un mot (graphie ou lemme). Le mot proposé par l'utilisateur est repéré dans le corpus et tous les paragraphes où on le trouve forment un texte composite qui est comparé à l'ensemble. Un calcul de spécificités met alors en valeur les mots qui sont significativement attirés par le mot-pôle et qui circonscrivent ainsi un «thème ». Ainsi les 5000 passages où l'on croise une fille dans le corpus Balzac (figure 1) montrent assez que son sort dépend de la famille et de la réponse qui sera donnée à la question essentielle : à qui la marier ?

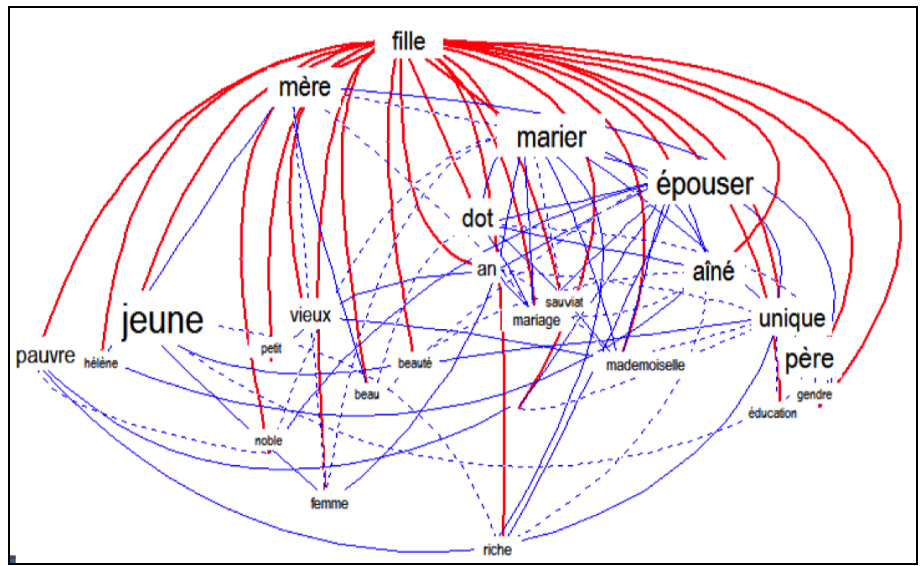

Figure 1. Le dessin d'un thème: autour du mot fille chez Balzac

Corpus $n^{\circ} 11$, La cooccurrence : du fait statistique au fait textuel (2012), 219-246 


\section{É. BRUNET}

1.2 On peut varier les questions et multiplier les thèmes, mais cette méthode, même cumulative, reste partielle et trop dépendante des choix proposés. On aimerait que le calcul seul délimite les thèmes exploités et construits dans le corpus, en interrogeant tous les mots sans en privilégier aucun. Pour ce faire, on devrait en principe croiser tous les vocables d'un texte et constituer un immense tableau qui aurait autant de lignes et de colonnes que de vocables recensés, soit une matrice de 100 millions de cases pour un petit corpus de 10000 mots. On préfère limiter l'exploration à un échantillon raisonné, en choisissant les mots les plus fréquents, ceux qui ont précisément le plus de chances de s'accoupler. On commence par constituer un tableau carré où les mots retenus (environ 300 parmi les mots-pleins les plus fréquents) sont portés à la fois dans les lignes et les colonnes; à l'intersection on a l'effectif des rencontres où le mot de la ligne i est en cooccurrence (dans le même paragraphe) avec le mot de la colonne j. Pour être plus précis, il faudrait parler de co-présence : quelle que soit la fréquence $f_{a}$ du mot $a$ et la fréquence $f_{b}$ du mot $b$ dans le paragraphe, la rencontre des deux mots est comptée pour 1. On pourrait aussi légitimement aligner la cooccurrence $\operatorname{sur} f_{a}$ ou $\operatorname{sur} f_{b}$, ou sur le produit $f_{a} * f_{b}$.

La matrice ainsi construite est ensuite soumise à l'analyse factorielle des correspondances, qui permet de visualiser les attirances entre les mots retenus.

Concrètement, pour établir avec le logiciel HYPERBASE la liste des mots à retenir, solliciter le bouton PREPARE. On recommande de se limiter aux substantifs, mais les verbes et les adjectifs sont aussi éligibles. Par défaut la sélection se fait d'abord sur la catégorie, puis sur la fréquence et enfin sur la taille du tableau. En aucun cas le nombre de mots retenus ne doit dépasser 400 (car, au-delà, trop de mots encombrant le graphique, les résultats perdent en lisibilité). Si ce nombre est dépassé lors d'un premier balayage, une seconde exploration, voire une troisième, est déclenchée automatiquement avec un seuil plus sévère. Prendre patience. Le programme est long, chaque paragraphe devant être examiné en séquence.

Quand le tableau des cooccurrences est constitué, l'exploitation statistique fait appel au programme d'analyse factorielle de correspondance LX3AFC.EXE écrit par Ludovic Lebart dans 


\section{Nouveau traitement des cooccurrences dans Hyperbase}

les années 70. Les résultats de l'analyse sont rapatriés et présentés par le bouton GRAPHIQUE, qu'on peut solliciter plusieurs fois, selon qu'on veut croiser les facteurs 1, 2 ou 3.

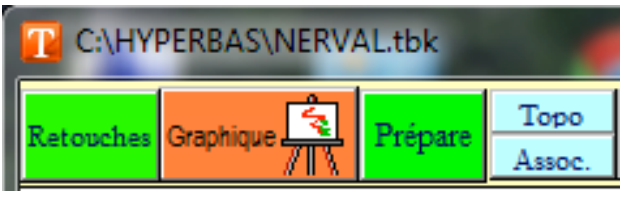

Il arrive parfois que des mots soient si souvent associés par la phraséologie, voire par le lexique (par exemple Etats et Unis) que cette liaison triviale déséquilibre le tableau et influence certains facteurs de l'analyse de façon excessive. On peut neutraliser cette influence gênante en intervenant dans le tableau des données et en diminuant la valeur observée au croisement de ces deux mots. Il arrive aussi que certains mots accaparent le pouvoir discriminant et qu'on souhaite réanalyser les données en dehors d'eux. Dans les deux cas le bouton RETOUCHES permet de modifier le tableau des données et de refaire l'analyse, sans nécessiter une nouvelle exploration du corpus. Cette exploration peut cependant être reprise si l'on sollicite le bouton PREPARE, qui montre à l'écran la liste enregistrée, si elle existe, et accepte l'effacement des éléments indésirables (un clic suffit).

Le tableau de données fournit les effectifs absolus des cooccurrences. L'analyse, quant à elle, s'appuie par défaut sur la racine carrée de ces données brutes, afin d'amortir l'effet de taille $^{1}$. Mais on peut renoncer à cette pondération et appliquer l'analyse factorielle aux effectifs absolus en sollicitant le bouton VARIANTES.

Ce même bouton VARIANTES propose une troisième option, qui fonde l'analyse sur des écarts réduits. Chaque cellule du tableau est alors soumise à un calcul probabiliste mettant en œuvre les lois normale et hypergéométrique.

1 Les données brutes enregistrées par le bouton PREPARE se trouvent dans le fichier qui porte le nom de la base suivi du suffixe .SOC. Les données converties en racine carrée se trouvent quant à elles dans le fichier qui porte le nom de la base suivi du suffixe. DON. 


\section{É. BRUNET}

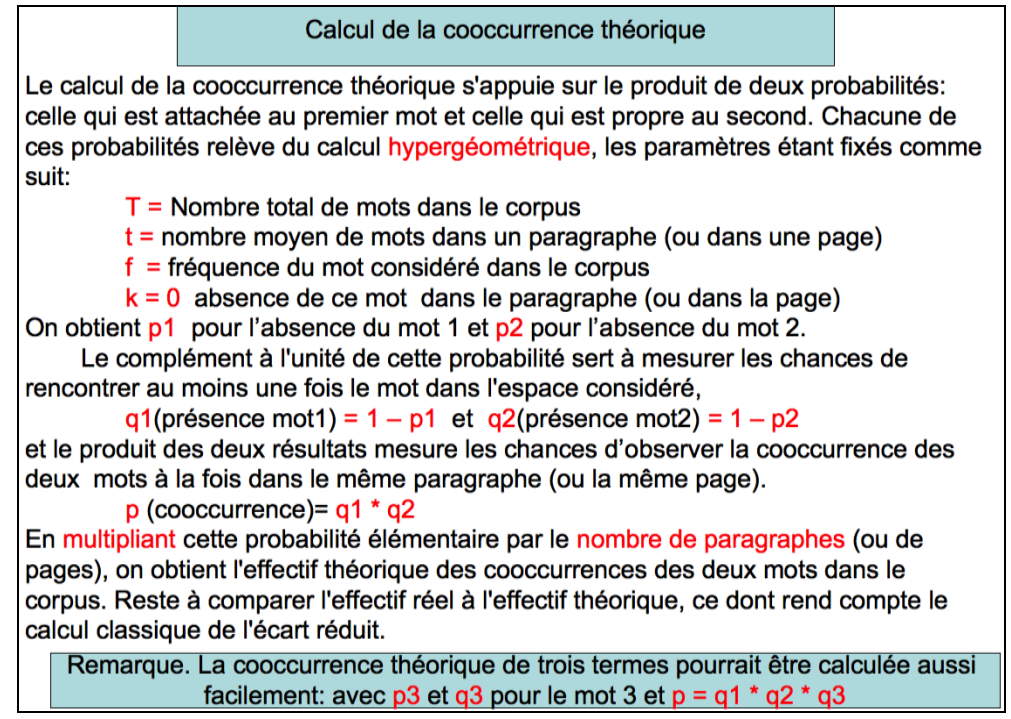

Figure 2. Calcul hypergéométrique de la cooccurrence théorique

On peut observer l'effet obtenu par l'option choisie dans VARIANTES en comparant les trois analyses factorielles qui peuvent être successivement réalisées à partir des mêmes données de départ. A première vue les différences paraissent faibles. Qu'il s'agisse d'effectifs bruts, ou convertis en racine carrée ou soumis au calcul probabiliste, les mêmes constellations lexicales s'ordonnent de la gauche à la droite, en allant du monde physique au monde moral (c'est le premier facteur). Dans les trois cas le facteur 2 oppose l'individu (en haut) à la société (en bas). Les noms qu'on peut donner à de telles constellations sont les mêmes et à la même place : ciel, eau, nature, habitat, corps, société, sentiment, droit, pensée, art. Voir figure 3 ci-dessous qui donne les résultats de l'analyse du corpus Nerval sur écarts réduits. 


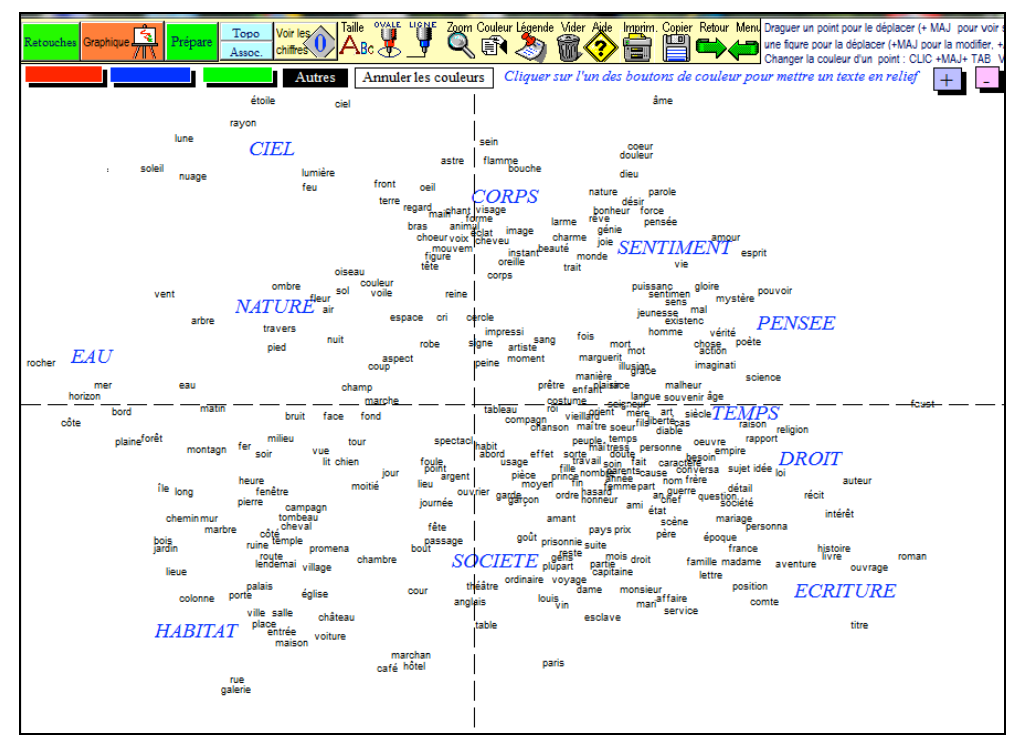

Figure 3. Analyse factorielle du corpus Nerval sur écarts réduits

Pourtant un examen attentif des trois analyses révèle des déplacements qui pour être de faible portée n'en sont pas moins systématiques. Ils tiennent à la manière de traiter les hautes et moins hautes fréquences. Quoique les substantifs réunis figurent parmi les fortes fréquences, puisque ce sont les 300 premiers de la catégorie, il peut y avoir de fortes inégalités en passant de la première à la dernière place. Le rapport est au moins de 1 à 10. On fera donc deux lots particuliers dans la population, en isolant les 50 mots les plus forts et parallèlement les 50 plus faibles. Pour opérer cette décantation, solliciter le bouton gris marqué d'un plus (les mots fréquents apparaissent en bleu) et le bouton rose marqué du signe moins (les moins hautes fréquences en rouge).

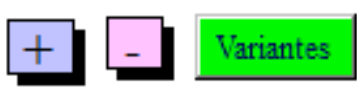




\section{É. BRUNET}

Tableau 1. Décantation des fréquences dans le corpus Nerval
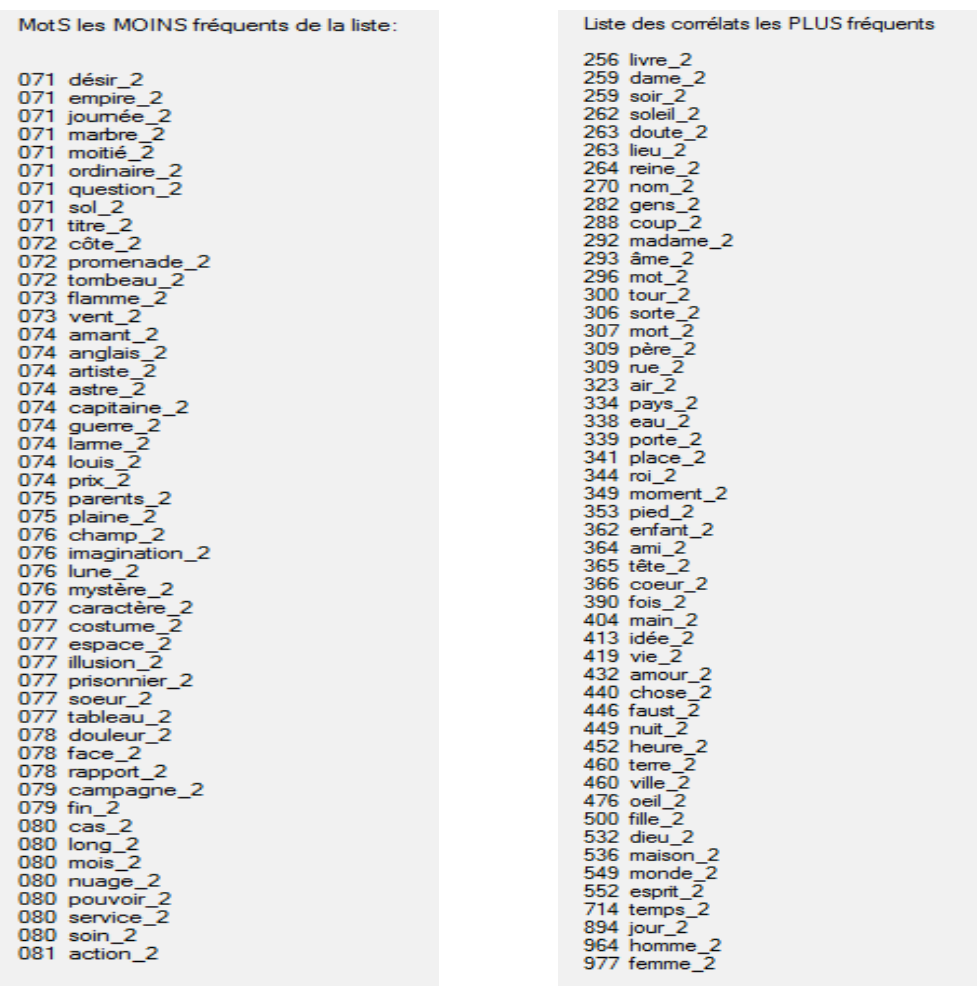

Pour plus de clarté on mettra dans l'ombre les autres mots (bouton AUTRES). Dès lors, on voit mieux le mouvement qui oriente vers le centre ou la périphérie les deux lots opposés. Quand les données sont brutes, les hautes fréquences se rapprochent du centre-ville où elles font la loi, en repoussant dans la banlieue les fréquences plus basses. C'est l'effet de taille classique. Sans doute la distance du CHI2, qui règne dans l'analyse de correspondance, tend à corriger ces déviances, mais elles restent visibles dans l'analyse ci-dessous (figure 4), qui rend compte des chiffres absolus. Les mots fréquents revêtus de bleu se concentrent près de l'origine des axes, tandis que les mots moins fréquents font un cercle rouge à l'entour.

Quand, au contraire, les inégalités trop fortes sont rabotées par la loi fiscale et hypergéométrique, la mixité et l'équilibre des classes tendent à se rétablir. C'est ce que montre la figure 5, 


\section{Nouveau traitement des cooccurrences dans Hyperbase}

fondée sur des calculs probabilistes (écarts réduits). Quant à l'analyse fondée sur les racines carrées des cooccurrences, elle propose un compromis qui atténue les inégalités, sans aligner les faits sur un lit de Procuste.

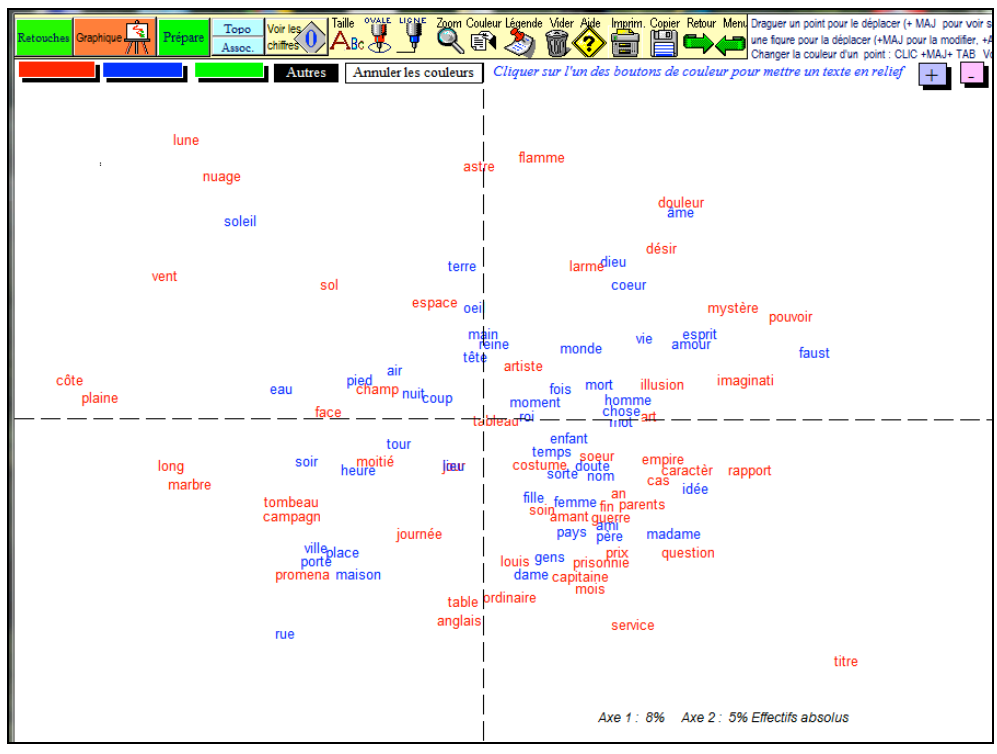

Figure 4. Cooccurrences brutes.

Les mots les plus fréquents se rapprochent du centre

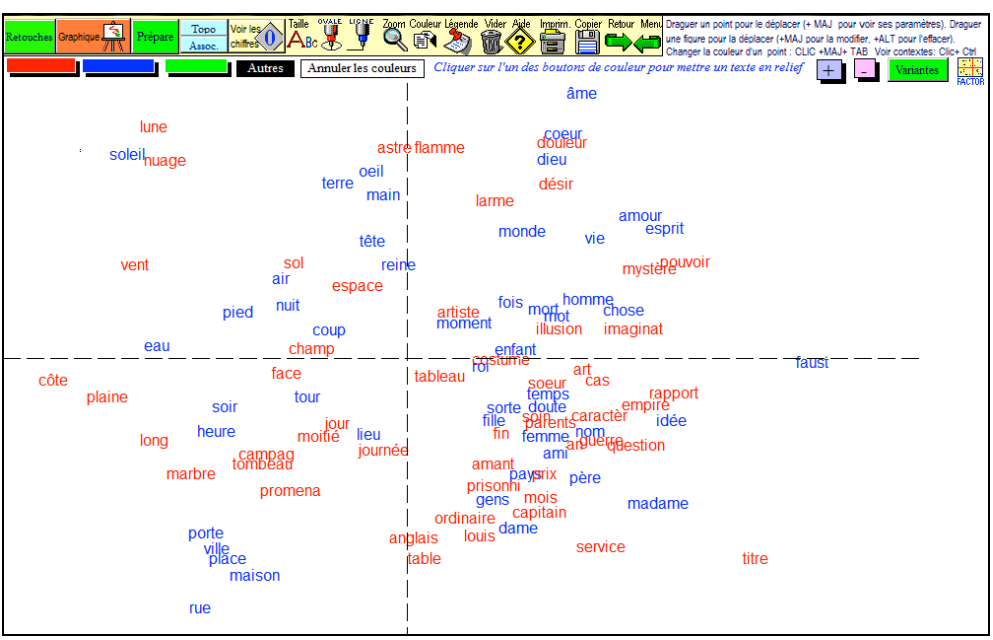

Figure 5. Ecarts réduits. Mixité et équilibre des deux classes 


\section{É. BRUNET}

Pour mettre en évidence le déplacement des populations, attachons-nous au lot des nantis et superposons les positions qu'ils occupent dans l'analyse brute (en rouge) et l'analyse réduite (en bleu). Ces positions sont toujours voisines et un même mot s'interdit de chevaucher un axe, vertical ou horizontal. Mais le décalage est constant pour chacun des mots : quand il apparaît en rouge, c'est-à-dire dans l'analyse brute, son poids le rapproche du centre par une sorte de gravité. Quand la péréquation est introduite, le même mot, représenté en bleu, est affranchi de la pesanteur et s'écarte de l'origine des axes. Si l'on représente parallèlement le lot des fréquences plus basses, le même mouvement est observé, mais inversé.

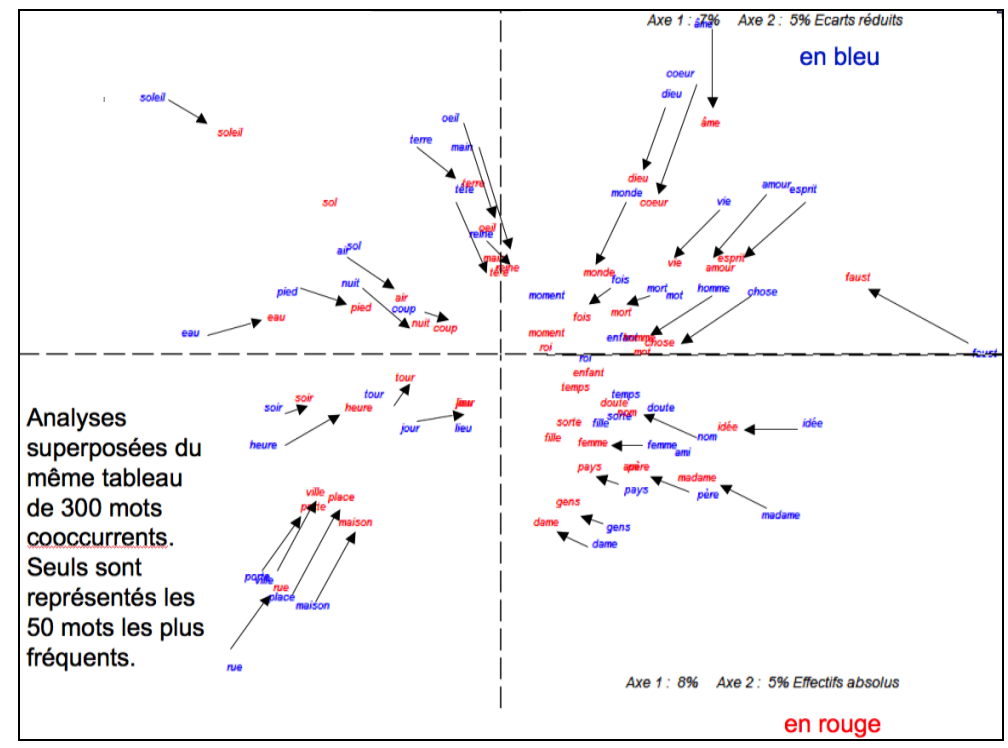

Figure 6. Les éléments lourds dans les analyses brute et réduite

1.3 Le réseau des cooccurrences ignore les barrières des textes et ne considère que le champ étroit du paragraphe. On peut cependant projeter l'image des textes sur le graphique en recourant aux boutons bleu, rouge et vert. On met ainsi en couleur les mots du graphique qui sont spécifiques d'un texte (ou de plusieurs). Faculté est donnée d'isoler ces spécificités en mettant dans l'ombre les autres mots, ou de les rétablir en effaçant ou non les couleurs. 
Nouveau traitement des cooccurrences dans Hyperbase

\begin{tabular}{|l|l|l|}
\hline \hline & Antres \\
\hline
\end{tabular}

Cliquer sur l'un des boutons de couleur pour mettre un texte en relief

La figure 7 ci-dessous reprend l'analyse de la figure 3 en évacuant tous les mots qui n'appartiennent pas aux spécificités des deux textes choisis. Le premier, relatif à Faust, s'installe en rouge dans le quadrant supérieur droit et se cantonne dans le vocabulaire des sentiments et des valeurs où se plaît le débat dramatique. Le second, relatif à Voyage en Orient, occupe l'espace opposé et multiplie les curiosités locales du tourisme culturel.

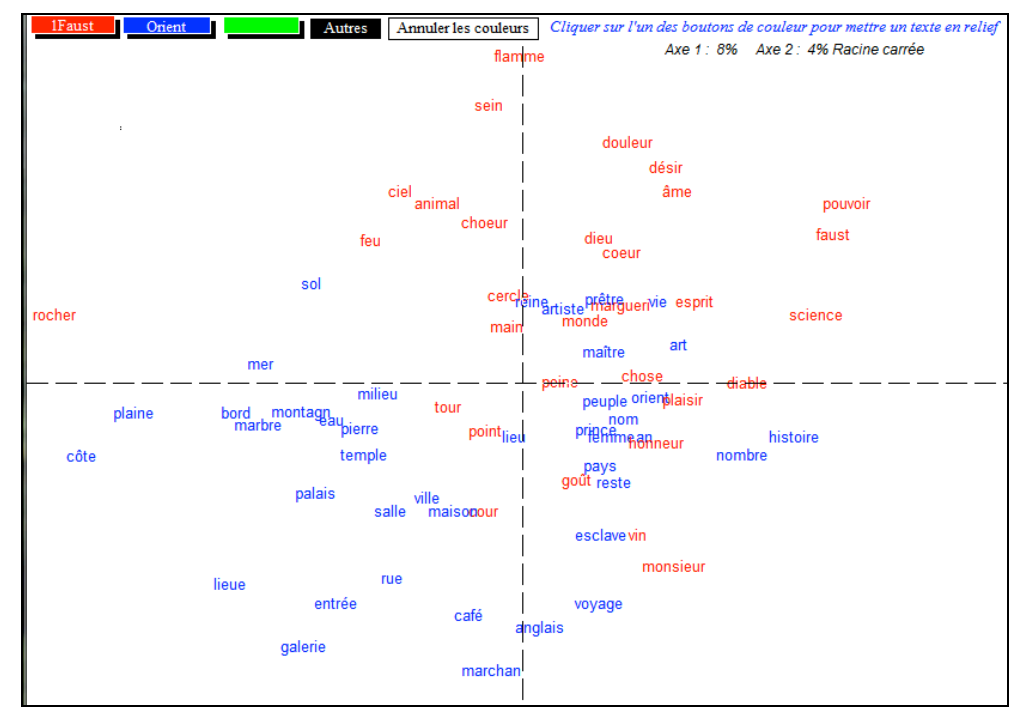

Figure 7. Textes et cooccurrences:

les choix opposés de Faust et du Voyage en Orient

\section{Cooccurrences et typologie textuelle}

Il est un moyen plus ambitieux de rétablir la séparation et la comparaison des textes, dans l'analyse même des cooccurrences. Le bouton FACTOR, à l'extrême droite, tente de répondre à un vœu exprimé par Mayaffre et Rastier : établir la typologie des textes non plus sur les occurrences des mots individuels, mais sur les cooccurrences qui lient les mots entre eux et sont porteurs du 


\section{É. BRUNET}

sens (le sens des mots n'étant pas dans le dictionnaire, dans la référence, mais dans la préférence, dans le contexte des mots associés).

\subsection{Nouvelle appréciation de la distance intertextuelle}

La difficulté vient du changement d'échelle. Un relevé de cooccurrences a trois dimensions: Ntextes * (Nmots * (Nmots-1)/2), là où un tableau d'occurrences n'en a que deux: Ntextes * Nmots. Notre approche est de se contenter des 300 substantifs les plus fréquents et d'ignorer les cooccurrences non observées. On tente d'imiter la démarche du logiciel ALCESTE, mais en empruntant un autre chemin. Au lieu de multiplier les cases vides d'un tableau encombrant, à trois dimensions, on établit une liste des cooccurrences réellement rencontrées. Un programme d'indexation et de tri s'exerce sur les relevés et en constitue un dictionnaire alphabétique, sous le nom de la base associé au suffixe .OCC (par exemple NERVAL.OCC). Un extrait de ce dictionnaire est présenté dans le tableau 2. Les cooccurrences apparaissent par couple en ordre alphabétique, avec leur fréquence dans le corpus, leurs adresses, et leur répartition dans les textes. Par exemple le couple abord_2_amant_2 est rencontré 2 fois dans le corpus, dont 1 fois dans le texte 4 et 1 fois dans le texte 5 .

Tableau 2. Extrait du dictionnaire des cooccurrences NERVAL.OCC

\begin{tabular}{|llllll|}
\hline abord_2_affaire_2 & 2 & 2056 & 2086 & 4 & 2 \\
\hline abord_2_âge_2 & 2 & 2891 & 3872 & 5 & 2 \\
\hline abord_2_air_2 & 1 & 4107 & 6 & 1 & \\
\hline abord_2_amant_2 & 2 & 2211 & 2785 & 4 & 1,5 \\
\hline abord_2_ame_2 & 1 & 1935 & 2 & 1 & \\
\hline abord_2_ami_2 & 1 & 3724 & 5 & 1 \\
\hline abord_2_amour_2 & 1 & 4559 & 8 & 1 \\
\hline abord_2_an_2 & 2 & 2032 & 2446 & 4 & 2 \\
\hline abord_2_animal_2 & 1 & 2080 & 4 & 1 \\
\hline etc. & & & & \\
\hline
\end{tabular}




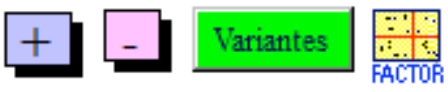

Le programme d'analyse factorielle (symbole ci-dessus) s'empare de ce dictionnaire, sans s'effrayer des milliers de lignes qu'il peut contenir (on peut écarter cependant les hapax et, dans les très gros corpus, les cooccurrences rares, limitées à quelques unités). Le fichier des paramètres de l'analyse réalisée sur Nerval, fait état de 15190 lignes différentes (tableau 3), dont les premières ont été montrées ci-dessus (tableau 2).

Tableau 3. Les paramètres de l'analyse factorielle des cooccurrences

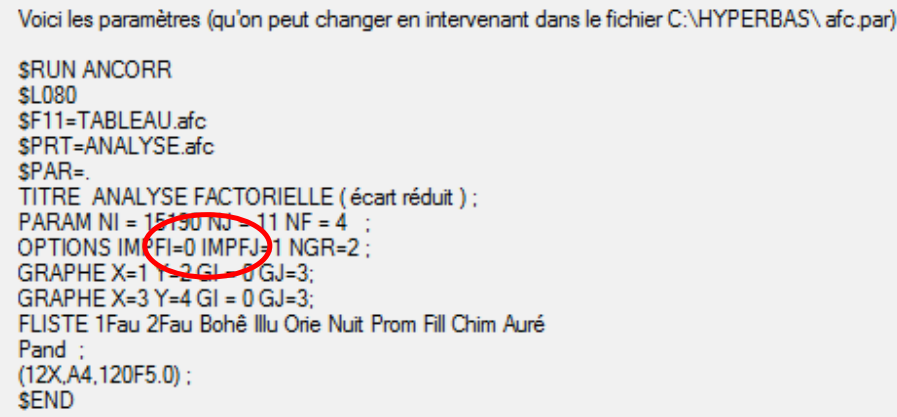

Les résultats sont consignés dans la figure 8 et confrontés à une analyse faite à partir des simples occurrences (figure 9). Noter que seuls les textes prennent position sur le graphique, les couples de mots en cooccurrence étant trop nombreux pour figurer sur l'écran. On pourrait toutefois les consulter en changeant les paramètres du programme ANCORR.EXE (fichier AFC.PAR) et en lisant les résultats dans le fichier ANALYSE.AFC. 
É. BRUNET

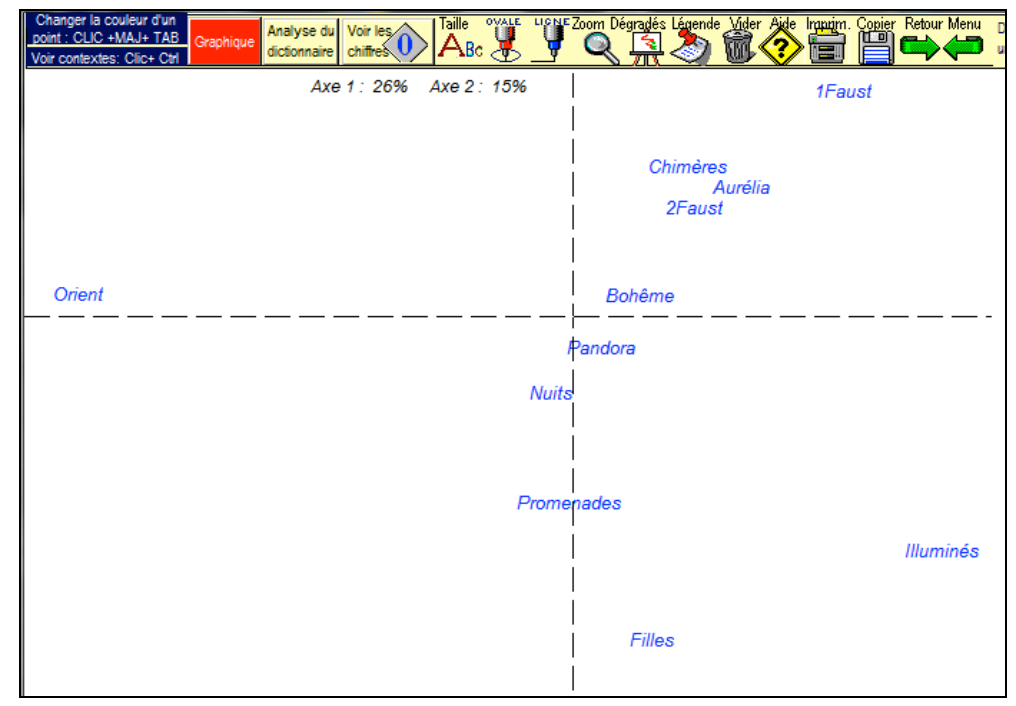

Figure 8. Analyse factorielle:

les textes de Nerval selon la distribution des cooccurrences

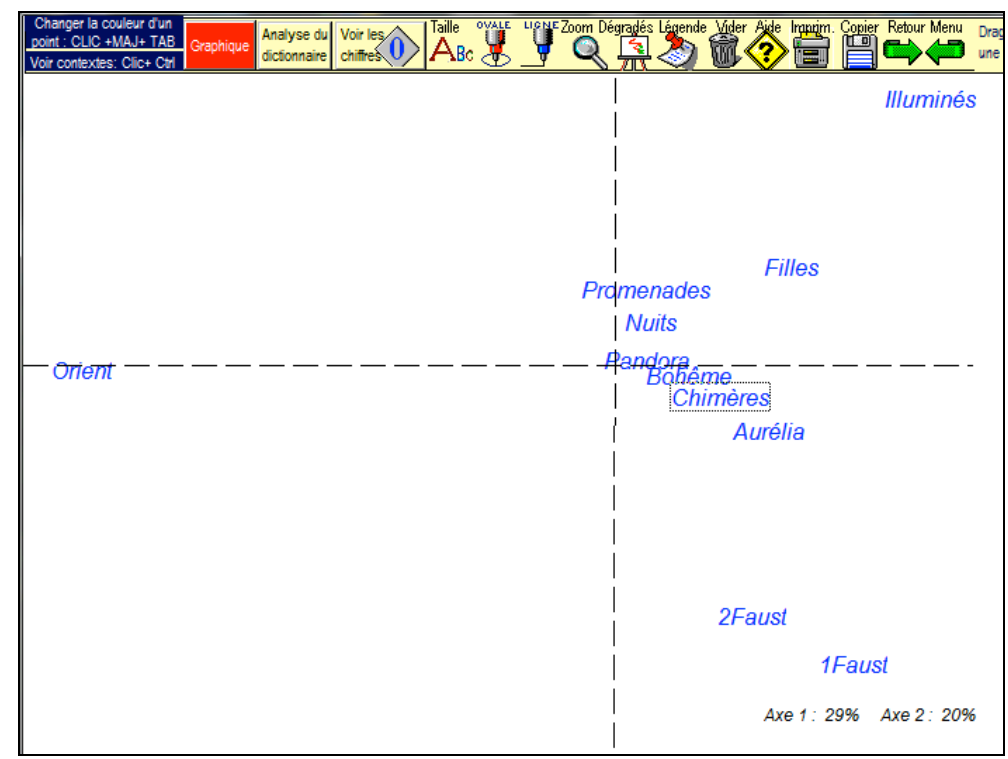

Figure 9. Analyse factorielle:

les textes de Nerval selon la distribution des occurrences 


\section{Nouveau traitement des cooccurrences dans Hyperbase}

Ce n'est pas le lieu ici pour développer des commentaires littéraires sur la typologie des textes nervaliens dont certains appartiennent à l'écriture de la description ou de l'évocation, comme le Voyage en Orient isolé à l'extrême gauche, d'autres à l'ordre dramatique (les deux Faust), ou poétique (Chimères) ou romanesque (Filles de feu, Illuminés).

Nous importe davantage la confrontation avec les résultats habituels qui portent sur les simples occurrences. Or, mis à part une inversion, sans réelle signification, qui change l'orientation verticale, la représentation que livrent les cooccurrences n'est que l'image en miroir de celle que produisent les occurrences dans la figure 9 , où les 2000 mots les plus fréquents sont pris en compte. Précisons que cette dernière analyse est obtenue par une option de la page FACTORIELLE, comme indiqué cidessous : dans les deux cas, on traite des profils, les fréquences brutes étant converties en écarts réduits.

\begin{tabular}{|c|c|c|}
\hline Graphique & $\begin{array}{l}\text { Analyse du } \\
\text { dicfonnaire }\end{array}$ & 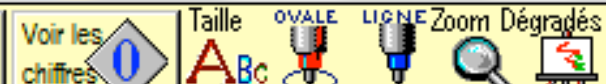 \\
\hline
\end{tabular}

C'est le cas aussi de l'analyse des 300 mots du tableau des cooccurrences, traités comme de simples occurrences. Une option du programme FACTOR y conduit. Ce tableau de 300 mots-lignes et de 11 textes-colonnes produit un graphique en tous points superposable au précédent, comme si ces 300 mots constituaient un échantillon admissible de la population.

Ce constat de la convergence est rassurant. On serait inquiet si des forces inconnues venaient à bouleverser l'ordre établi. Mais un brin de frustration accompagne et affadit la satisfaction. On pouvait rêver d'une lexicométrie quantique qui contesterait les observations et les lois de la lexicométrie traditionnelle. De même, dans le passé, l'accès plus récent au lemme aurait pu affaiblir, voire contredire, le traitement des graphies. Il n'en fut rien. Ainsi, en s'élevant des graphies aux lemmes, et des occurrences aux cooccurrences, la construction lexicométrique, d'étage en étage, reproduit les mêmes structures et s'appuie sur les mêmes fondations. Cela est même vrai quand on suit le chemin inverse et qu'on s'enfonce dans l'infralexical : on a démontré que la typologie des textes se maintenait 


\section{É. BRUNET}

inchangée quand elle portait sur les n-grammes ou séquences de 4 lettres, et qu'elle survivait ainsi au dépeçage des mots et à la ruine de la syntaxe et de la sémantique. Mieux ou pire encore, elle résiste même à la mort de l'alphabet: car l'ossature des textes reste parfaitement visible quand le texte, réduit en cendres, n'a plus qu'un code à trois éléments : voyelle, consonne et blanc.

\subsection{Les corpus}

Dans un dernier effort, grimpons d'un étage encore. Cette fois le panorama s'étend non plus sur la chaîne orientée des textes, mais sur des massifs indépendants, encore plus larges : les corpus. Il va sans dire que le survol simultané de ces corpus implique qu'ils aient été soumis aux mêmes traitements et que chaque base dispose d'un fichier où sont consignés les tableaux de cooccurrences. Un tel fichier porte le nom de la base et une finale en ".SOC », à l'image de celui qui accompagne la base Nerval et dont un extrait a été présenté précédemment dans le tableau 2.

Comme la liste des 300 mots cooccurrents n'est pas identique dans toutes les bases, chacune faisant son choix indépendamment des autres, le traitement est plus épineux que dans le cas simple d'une base unique. Il convient donc de passer en revue les différents corpus qu'on peut comparer à partir des relations cooccurrentielles.

La chiquenaude initiale part du bouton FACTOR, qui propose de choisir le niveau de l'enquête, entre textes et corpus.

Que voulez-vous faire?

Croiser les COOCCURRENCES et les TEXTES Croiser les COOCCURRENCES et les CORPUS

Si l'option CORPUS est choisie, s'ensuit une invitation à choisir les différents corpus, rassemblés dans le répertoire $\mathrm{C}: \backslash \mathrm{HYPERBAS} \backslash$. On utilisera la souris associée à la touche CTRL pour épingler dans le même mouvement les fichiers à suffixe .SOC qu'on veut retenir parmi ceux qui sont disponibles. Lorsque le choix est fait, il durera ce que dure la séance en cours, avec cependant la possibilité de le modifier. Dans la suite du traitement, l'ordre alphabétique de ces fichiers sera respecté. S'il ne convient pas, ajouter préalablement un numéro 
Nouveau traitement des cooccurrences dans Hyperbase

d'ordre devant les noms des fichiers à traiter pour imposer le classement désiré.

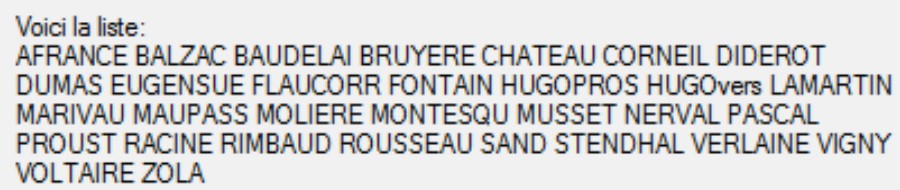

On procède alors à l'exploration des différentes bases ou plus précisément du fichier où est enregistré le détail des cooccurrences qu'on y trouve. De tableaux remplis de chiffres on tire un long ruban de cooccurrences, répétées autant de fois que nécessaire, avec pour seule structure un jalon indiquant le passage d'un corpus à l'autre. En somme on a constitué un texte réduit aux seules cooccurrences, lesquelles seront considérées comme des mots ordinaires une fois qu'on a collé l'un à l'autre les deux membres de la cooccurrence. Dès lors les programmes habituels d'indexation peuvent être mis en œuvre, pour aboutir à un dictionnaire récapitulatif des cooccurrences, pourvu des sous-fréquences par corpus (Tableau 4).

Tableau 4. Extraction et regroupement des cooccurrences à partir de plusieurs corpus

$\& \& \& 1,1,01 \& \& \&$ abbé_2_abeille_2 abbé_2_abord_2 abbé_2_action_2 abbé_2_action_2 abbé_2_affaire_2 abbé_2_affaire_2 abbé_2_affaire_2 abbé_2_affaire_2 abbé_2_ami_2 abbé_2_âge_2 abbé_2_air_2 abbé_2_air_2 abbé_2_air_2 abbé_2_âme_2 abbé_2_âme_2 abbé_2_ami_2 abbé_2_ami_2 abbé_2_amour_2 etc. abbé_2_âge_2 abbé_2_air_2 abbé_2_air_2 abbé_2_âme_2 abbé_2_âme_2 abbé_2_âme_2 abbé_2_ami_2 abbé_2_amour_2 


\section{É. BRUNET}

Poursuivant son chemin, le programme explore chaque ligne de ce dictionnaire des fréquences et soumet l'ensemble à l'analyse factorielle. Les données étant des effectifs bruts, démunis de probabilités, il n'est guère envisageable d'en tirer des écarts. Afin de limiter l'effet de taille qu'on peut craindre avec des corpus et des mots de poids inégal, les données brutes sont converties en racine carrée. Le résultat apparaît dans la figure 10.

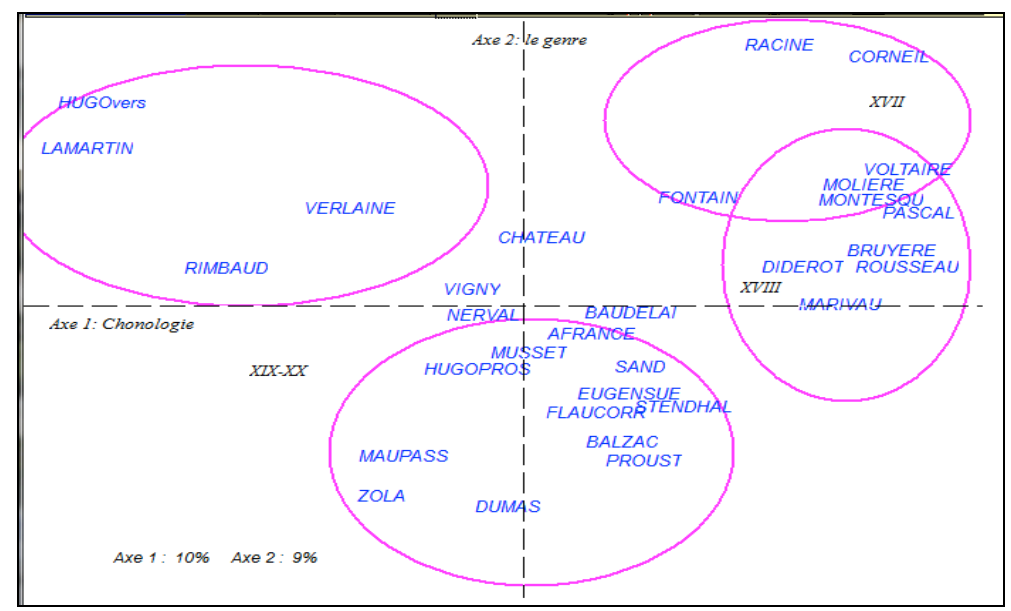

Figure 10. Analyse factorielle de plusieurs corpus selon la distribution des cooccurrences

On a juxtaposé les résultats obtenus avec les mêmes données, préalablement dégroupées. Les mots qui entrent dans la composition de la cooccurrence sont maintenant traités individuellement, comme de simples occurrences. Dans l'urne s'agitent des mots indépendants qui se considèrent comme étrangers les uns aux autres, une révolution morale ayant aboli toutes les unions ou accordailles. Ce traitement est assuré par une option du bouton FACTOR (figure 11).

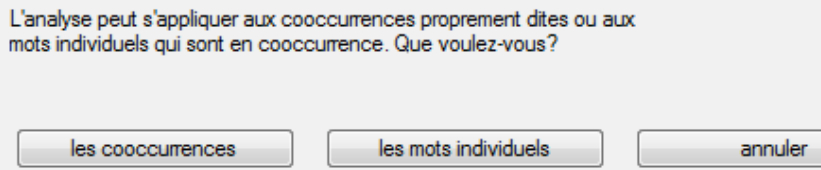




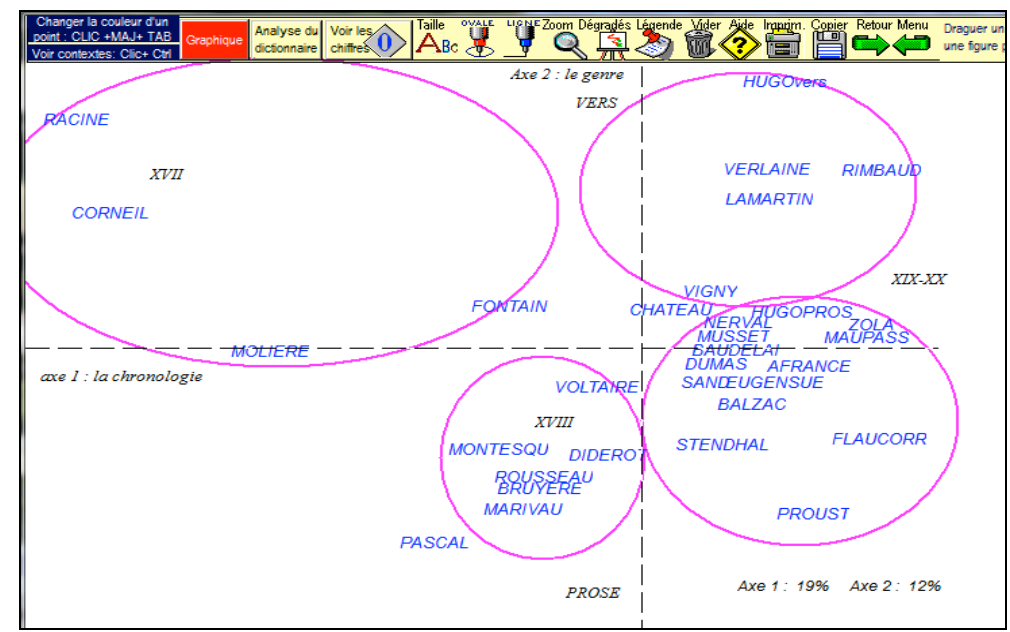

Figure 11. Analyse factorielle de différents corpus selon

les occurrences simples, après dégroupement des cooccurrences

On n'attachera aucune signification à l'inversion qui présente en miroir le premier facteur, qui dans les deux cas est le reflet de la chronologie : d'un côté le XVII siècle et le XVIII ${ }^{\mathrm{e}}$, de l'autre le $\mathrm{XIX}^{\mathrm{e}}$ et le $\mathrm{XX}^{\mathrm{e}}$. Pareillement dans les deux figures le second facteur rend compte du genre, les vers à la surface, la prose en profondeur. Mais un brouillage s'observe parallèlement dans les deux analyses : l'interférence du genre dans la chronologie. La Bruyère et Pascal se détachent des versificateurs du siècle classique pour rejoindre les prosateurs moralistes du $\mathrm{XVIII}^{\mathrm{e}}$. Molière, dont l'œuvre est pour la moitié en prose, subit cette attirance, tandis que Voltaire, à cause de son théâtre versifié, s'oriente dans le sens inverse. La production multi-genre n'est pas l'apanage de Voltaire. Elle est observée chez beaucoup d'écrivains comme Vigny, Musset, Nerval, Chateaubriand ou Baudelaire, qui divisés et indécis se rapprochent par là même du marais central. Les positions seraient plus nettes si la séparation introduite chez Hugo entre les œuvres en vers et les œuvres en prose avaient été généralisée. Mais le genre implique bien d'autres catégories que le vers et la prose, et la diversification des données pouvait s'étendre à l'infini.

Mais comme précédemment le but n'est pas d'obtenir une carte fidèle de la littérature française mais de vérifier si 


\section{É. BRUNET}

l'approche par les cooccurrences plutôt que par les occurrences apporte une précision supplémentaire, voire une vision renouvelée. La réponse semble devoir être provisoirement évasive, les deux analyses étant superposables. On se gardera d'en conclure que l'examen des cooccurrences est inutile : une confirmation n'est jamais une épreuve superfétatoire. On s'en convaincra avec l'examen du théâtre classique (figure 12). Pratiqué sur les cooccurrences, il reproduit exactement l'image obtenue sur les occurrences : une carte où les trois écrivains délimitent nettement leur territoire, sauf dans les rares occasions où les lois du genre s'y opposent (les Plaideurs de Racine au milieu des pièces de Molière, la pièce sérieuse de Molière, Garcie de Navarre, parmi les tragédies de Corneille).

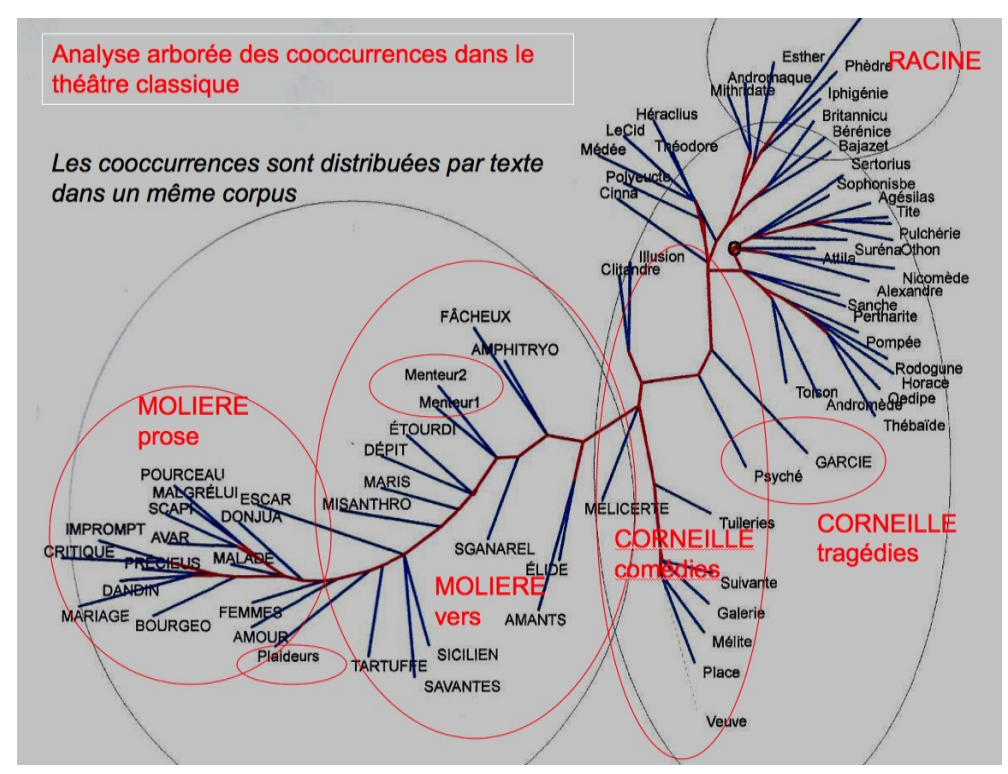

Figure 12. La distance intertextuelle, calculée sur les cooccurrences

\subsection{Cooccurrences et réseaux thématiques}

Mais si l'image globale n'est pas bouleversée, le détail de la distribution apporte souvent des indications lumineuses sur telle ou telle thématique. Pour illustrer cette approche dans Hyperbase, on doit activer le bouton ASSOCIATIONS qui voisine avec le bouton CORRELATS dans le menu principal. En réalité ces 
Nouveau traitement des cooccurrences dans Hyperbase

deux dénominations recouvrent la même chose : les cooccurrences. Mais avec les «corrélats» on envisage l'aspect global du phénomène dans le cadre d'une sélection représentative, et avec les "associations », même "généralisées », on privilégie leur étude détaillée, dans le même cadre. Quand la page ASSOCIATIONS invite à "choisir un pôle», proposons par exemple le mot bonheur.

Parmi les 300 mots de la sélection, le bonheur a la bonne fortune de trouver des alliés (parmi lesquels même des antonymes). Or un lien peut être établi avec les autres bases disponibles, pour observer si les relations cooccurrentielles y sont semblables ou différentes. Le bouton HISTOGRAMME, non content de reproduire sur un graphique les relations préférentielles du mot-pôle dans le corpus exploré, invite à y ajouter d'autres corpus, au moins ceux qui ont des données comparables à propos du mot considéré. Ainsi voit-on dans le graphique ci-dessous (figure 13) que chez Pascal le bonheur est dans l'au-delà et dans un rapport à Dieu tandis que Proust y voit un rêve impossible associé au chagrin, à la souffrance, au temps destructeur, à la tristesse et bien sûr au désir et à l'amour. Si l'on juxtaposait les données de Stendhal, grand assoiffé de bonheur, l'amour serait aussi au premier plan, mêlé à l'ennui, à l'orgueil et à la vanité.

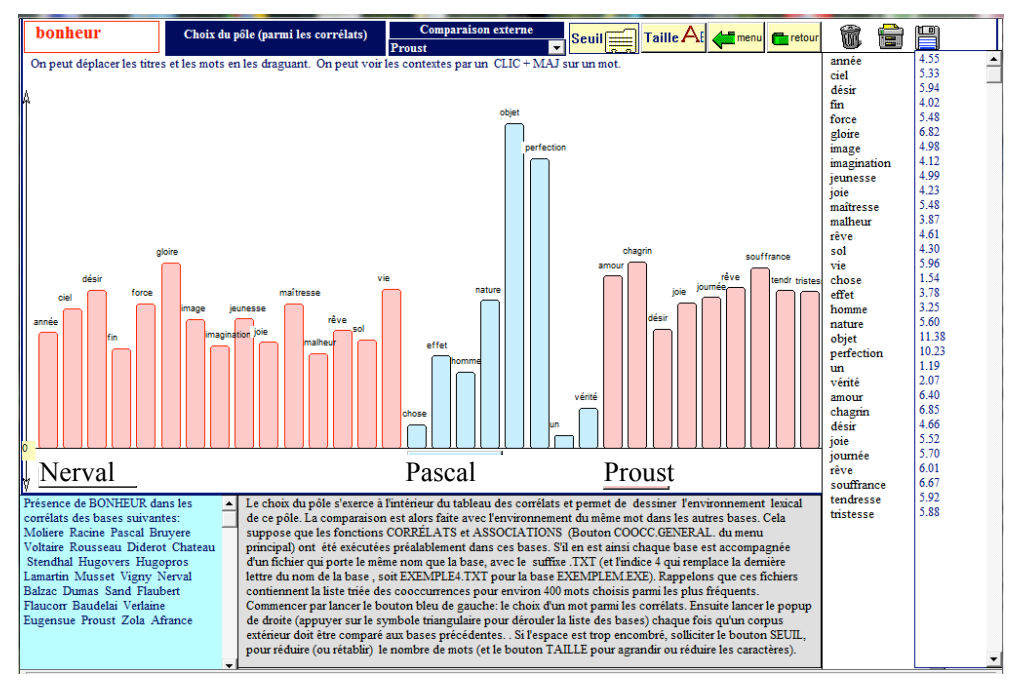

Figure 13. L'environnement du bonheur chez quelques écrivains 


\section{É. BRUNET}

Cette excursion des cooccurrences hors du corpus semble très riche d'information et renouvelle l'approche comparative. Si l'on se contentait des occurrences simples relevées sans tenir compte du voisinage, la courbe du bonheur serait beaucoup plus pauvre et sèche, sans les connotations ou harmoniques que permet l'environnement cooccurrentiel. Le graphique de la figure 14 a beau répertorier 14000 emplois du mot chez 75 écrivains, on voit bien que Stendhal est le champion de la catégorie, mais la notion de bonheur n'y reçoit aucun éclairage. Même sans polysémie trop violente, le mot reste enveloppé dans un brouillard que la cooccurrence aiderait à percer.

\subsection{Spécificités cooccurrentielles}

La tentative précédente est limitée à un zoom spectaculaire mais instantané, qui n'éclaire qu'un mot à la fois. C'est aussi le reproche qu'on peut faire à l'entreprise de Google Books, qui a canalisé les textes de l'édition mondiale dans un réservoir monstrueux, dont 44 milliards de mots pour le domaine français! Mais à la sortie de cet immense bassin, il n'y a qu'un robinet étroit qui distribue les mots un par un, au compte-gouttes. C'est pourquoi en puisant dans le même bassin (généreusement téléchargeable), on a cru devoir constituer une base plus ouverte (appelée GOOFRE) où puissent s'employer les outils de la statistique et notamment les analyses multidimensionnelles.

Ne pourrait-on pas tenter une expérience semblable avec les cooccurrences? Damon Mayaffre dans un colloque tenu à Rome en octobre 2011 regrettait qu'il n'existe pas pour les cooccurrences une méthodologie lexicométrique analogue à celle qui a cours pour les occurrences. A priori on pourrait estimer que c'est la même, mais à une autre échelle, la seconde étant de l'ordre du carré de la première (plus précisément à la dimension $n$ correspond la dimension $n *(n-1) / 2)$. Deux obstacles liés se dressent sur la route: d'une part la taille démesurée des tableaux et la longueur des calculs, et d'autre part la faiblesse des effectifs, la plupart étant nuls. Pour échapper à ce dilemme, on propose de diminuer le nombre de mots étudiés, en réponse à la première aporie, et d'augmenter la taille des données, pour répondre à la seconde. La solution a été entrebâillée dans les pages qui précèdent : ne s'intéresser qu'à une fraction restrictive mais représentative de la population, les 300 substantifs les 


\section{Nouveau traitement des cooccurrences dans Hyperbase}

mieux représentés dans chaque corpus. Et parallèlement étendre l'enquête à tous les corpus qu'on voudra comparer.

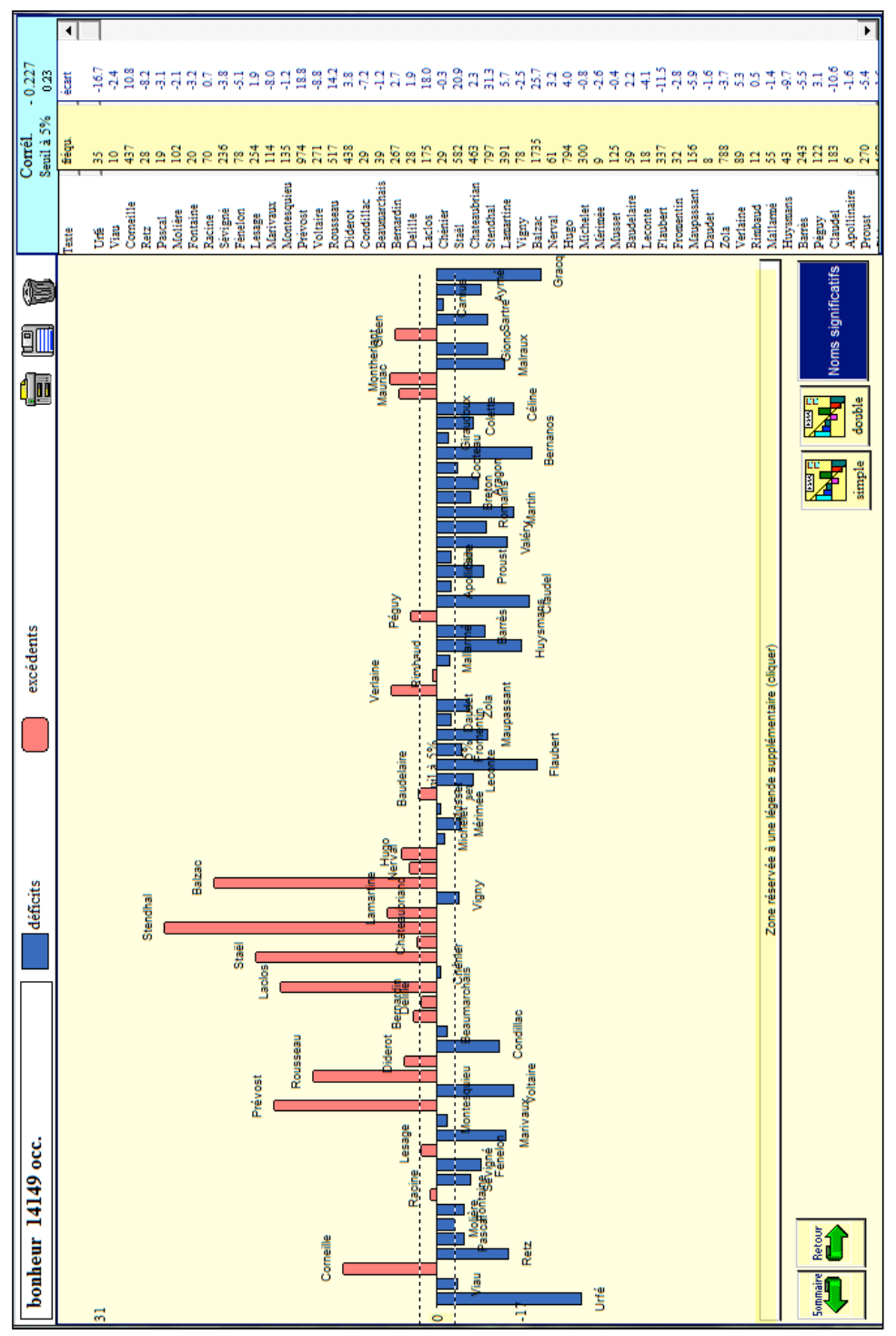

Figure 14. Le bonheur sans assaisonnement. Répartition chez 75 écrivains

Pour n'être pas lié à une fonction particulière d'un corpus particulier, on a créé une version nouvelle d'Hyperbase, 


\section{É. BRUNET}

dénommée HYPoccur. C'est un modèle riche de programmes et vide de données, comme Hypertag ou Hypercor. Quand on le lance, il génère une copie que l'on est invité à remplir. Il ne demande qu'une seule espèce de données : non pas des textes, mais des tableaux de cooccurrences, expressément consignés par Hyperbase dans des fichiers à suffixe .SOC. La procédure initiale est donc la même que celle qu'on a décrite précédemment (tableau 4). Mais le traitement diverge ensuite. Au lieu de créer seulement un dictionnaire des fréquences, on constitue une véritable base, semblable à celles qu'on peut réaliser avec Hyperbase. La suite des mots en cooccurrence est enregistrée à la queue-leu-leu, comme s'il s'agissait d'un véritable texte en continu. Quand on passe d'un corpus à l'autre, un jalon s'interpose, qui joue le même rôle que la barrière qui sépare deux textes dans un corpus normal. Dès lors tout s'accomplit jusqu'au terme sans changement notable dans le traitement, les couples cooccurrents devenant des mots et les corpus des textes.

Une fois la base constituée, les fonctions documentaires et statistiques d'Hyperbase sont accessibles, même si certaines perdent leur justification, comme la "concordance» ou la « topologie », vu le statut particulier des données. On peut certes renouveler le calcul de l'analyse factorielle, présentée dans le graphique 12, mais beaucoup d'autres, sur choix partiel ou différent, peuvent présenter de l'intérêt. La page Liste offre toutes les options possibles tant pour la sélection des données que pour le choix des outils. Parmi ceux-ci les figures 15 et 16 offrent deux variétés de représentation arborée, l'une fondée sur la «connexion » de Muller, l'autre sur la formule d'Evrard. Dans les deux cas, il s'agit d'apprécier la distance intertextuelle à partir des relations cooccurrentielles. Dans les deux graphiques, des régions périphériques au contour net, circonscrites autour de corpus homogènes (XVII ${ }^{\mathrm{e}}, \mathrm{XVII}^{\mathrm{e}}$, Roman, Vers) enveloppent une zone centrale, plus floue, où le mélanges des genres crée l'indécision.

A côté des vues synthétiques, factorielles ou arborées, des faits saillants peuvent sortir d'un simple histogramme, comme celui du couple mère-enfant, que les siècles classiques semblent ignorer, jusqu'à ce que Rousseau s'indigne et s'enflamme dans l'Emile à propos de l'allaitement. Tous ceux qui l'ont précédé dans la littérature s'inscrivent dans la zone né- 


\section{Nouveau traitement des cooccurrences dans Hyperbase}

gative. Les valeurs de la maternité et de l'enfance se déploient avec et après Rousseau dans la génération qui, de Diderot à Balzac, a eu à souffrir des nourrices et des internats. On observera que, sous ses airs de garçonne, Georges Sand trône au sommet de la courbe de la figure 17, comme symbole non seulement de la féminité mais de la maternité.

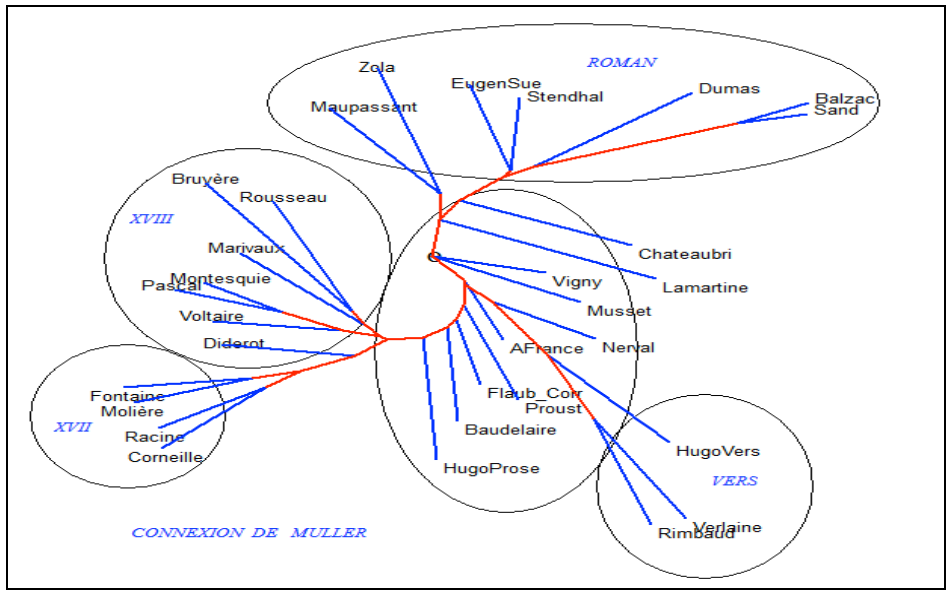

Figure 15. Analyse arborée selon la « connexion » de Muller

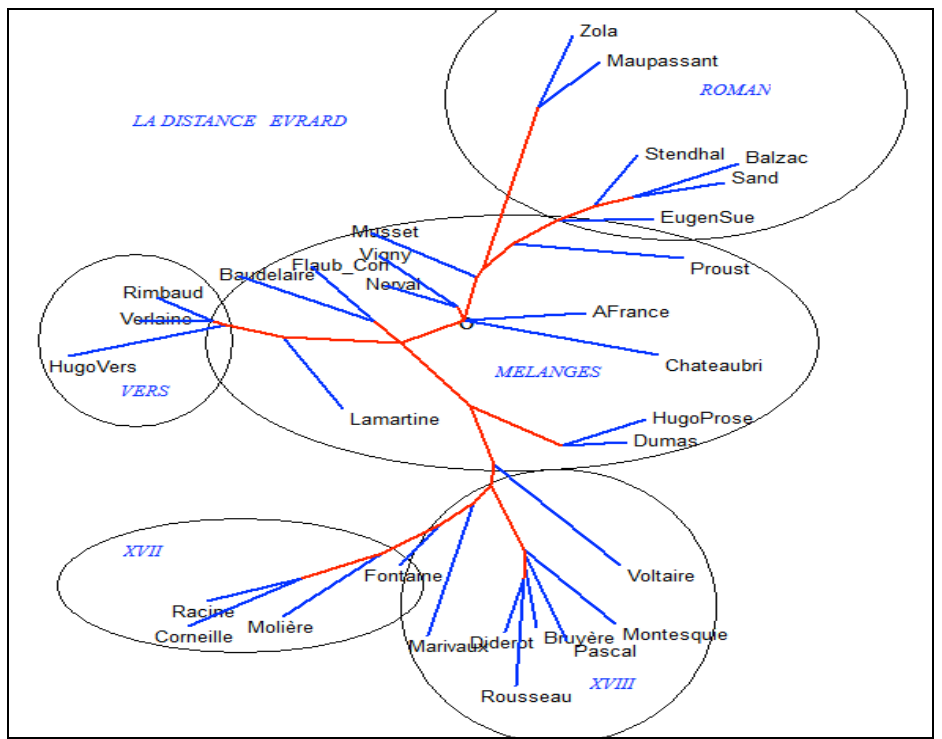

Figure 16. Analyse arborée selon la formule d'Etienne Evrard 
É. BRUNET

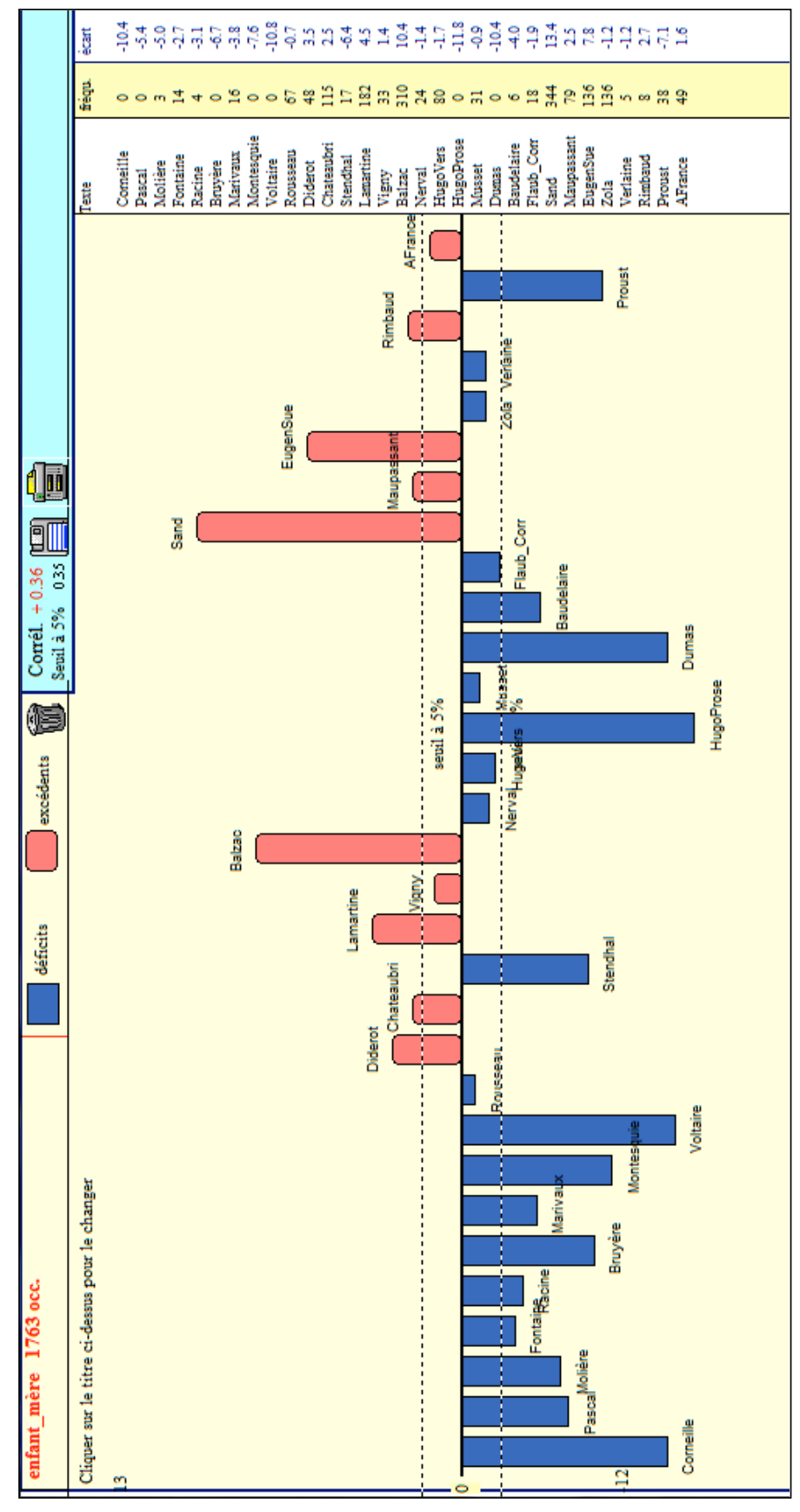

Figure 17. Graphique du couple mère-enfant 


\section{Nouveau traitement des cooccurrences dans Hyperbase}

L'évolution des mœurs qu'on touche du doigt à propos d'un couple comme mère-enfant ou travail-famille, peut être appréhendée globalement à partir du coefficient de corrélation, calculé pour tous les couples. Ceux qui gagnent ou perdent en crédit apparaissent dans le tableau 5.

Tableau 5. L'évolution des couples lexicaux. En progression (à gauche) et en régression (à droite)

\begin{tabular}{|c|c|c|c|c|c|c|}
\hline \multicolumn{4}{|c|}{ Progression Fréquence Forme } & \multicolumn{3}{|c|}{ Régression Fréquence Forme } \\
\hline+0.672 & 137 & air_travers & $\Delta$ & -0.627 & 81 & gloire_lieu \\
\hline+0.666 & 301 & air_soir & & -0.626 & 107 & coeur_discours \\
\hline+0.609 & 167 & année_heure & & -0.616 & 166 & oeil_vertu \\
\hline+0.602 & 286 & air_fleur & & -0.609 & 176 & jour_vertu \\
\hline+0.602 & 235 & air_matin & & -0.605 & 162 & âme_oojjet \\
\hline+0.595 & 143 & fleur_heure & & -0.603 & 76 & état_honneur \\
\hline+0.591 & 319 & chambre_nuit & & -0.602 & 170 & lieu_roi \\
\hline+0.583 & 308 & chambre soir & & -0.598 & 75 & faveưr grâce \\
\hline+0.582 & 143 & soir_tāble & & -0.586 & 300 & esprit_lieu \\
\hline+0.582 & 107 & côté_soir & & -0.585 & 396 & coeur_vertu \\
\hline+0.579 & 102 & face_monde & & -0.585 & 101 & faveur_homme \\
\hline+0.575 & 101 & douté nuit & & -0.584 & 139 & gloire-vertu \\
\hline+0.573 & 237 & chambre_milieu & & -0.583 & 86 & chose_pouvoir \\
\hline+0.569 & 158 & heure_joie & & -0.579 & 191 & honneur_vertu \\
\hline+0.566 & 142 & saint_soir & & -0.575 & 105 & coeur_fäveur \\
\hline+0.565 & 155 & année fille & & -0.573 & 125 & coeur-pouvoir \\
\hline $\begin{array}{l}+0.564 \\
+0.564\end{array}$ & 554 & chambre_lit & & -0.572 & 118 & honneur_lieu \\
\hline+0.563 & 230 & fenêtre_nuit & & -0.572 & 114 & âme_ordre \\
\hline+0.563 & 92 & eau_facē & & -0.570 & 138 & âme_soin \\
\hline+0.562 & 117 & chambre_lumière & & -0.568 & 91 & cour_lieu \\
\hline $\begin{array}{l}+0.562 \\
+0.52\end{array}$ & 93 & bonheur rêve & & -0.562 & 98 & honneur intérêt \\
\hline+0.560 & 307 & mère_soír & & -0.562 & 83 & coeur_ennemi \\
\hline+0.560 & 91 & mère_table & & -0.558 & 79 & âme_bien \\
\hline+0.557 & 111 & chambre_fleur & & -0.554 & 107 & coeur_cour \\
\hline+0.556 & 113 & fille_souvenir & & -0.553 & 171 & force_vertu \\
\hline+0.554 & 88 & regard_table & & -0.553 & 131 & amant_moment \\
\hline $\begin{array}{r}+ \\
+0.554\end{array}$ & 84 & année_doute & & -0.550 & 166 & coeur soupir \\
\hline+0.553 & 352 & air_pied & & -0.550 & 95 & bien_honneur \\
\hline+0.553 & 155 & matin_tête & & -0.548 & 95 & jour_pouvoir \\
\hline+0.552 & 123 & pied_table & & -0.547 & 252 & esprit_peine \\
\hline+0.552 & 114 & air argent & & -0.546 & 145 & bien coeur \\
\hline+0.551 & 237 & heure travail & & -0.545 & 240 & coeur objet \\
\hline+0.551 & 82 & doute soir & & -0.545 & 85 & coeur mérite \\
\hline
\end{tabular}

Ce faible extrait d'une liste beaucoup plus longue montre assez la tendance littéraire qui s'éloigne des abstractions un peu laiteuses dont s'abreuvaient les siècles classiques: la vertu, l'honneur, la gloire, l'âme, la grâce, le cour, et même l'esprit, qui se mêlent à loisir dans les combinaisons cooccurrentielles de l'époque, voient leur cours dévalué dans la colonne des régressions. Inversement la colonne de gauche souligne l'invasion du concret, du cadre de vie (chambre, fenêtre, lit, table, fleur, eau, lumière), du corps humain (pied, tête, regard), des relations de famille (mère, fille) et surtout l'obsession du temps : sur les 32 couples les plus prisés de notre époque, le soir compte 6 mentions, l'heure 4, la nuit 3, le matin 3 et l'année 2. 


\section{É. BRUNET}

Tableau 6. Les spécificités cooccurrentielles de Corneille et de Rousseau

\begin{tabular}{|c|c|c|c|}
\hline \multicolumn{4}{|c|}{ Cliquer sur un mot pour connatre sa spéciticité dans le } \\
\hline \multicolumn{4}{|c|}{$\begin{array}{l}\text { Cliquer sur un mot pour connatre sa spécificité dans le } \\
\text { écart corpus texté mot }\end{array}$} \\
\hline \\
\hline \multicolumn{4}{|c|}{$\begin{array}{llll}25.3 & 704 & 200 & \text { état_horme } \\
22.3 & 231 & 109 & \text { auteur livre }\end{array}$} \\
\hline \multirow{2}{*}{$\begin{array}{l}20.7 \\
19.6\end{array}$} & 230 & 101 & état_nāture \\
\hline & 50 & 50 & autrūi_horme \\
\hline \multirow{2}{*}{$\begin{array}{l}18.0 \\
17.8\end{array}$} & 92 & 60 & corps membre \\
\hline & 64 & 51 & éducation_horme \\
\hline \multirow{2}{*}{$\begin{array}{l}17.1 \\
16.8\end{array}$} & 317 & 95 & devoir_hơme \\
\hline & 81 & 53 & citoyen_droit \\
\hline & 618 & 127 & besoin_horme \\
\hline & 129 & 63 & droit_nature \\
\hline \multirow{2}{*}{$\begin{array}{l}16.0 \\
15.9\end{array}$} & 72 & 48 & corps_volonté \\
\hline & 118 & 58 & citoyen_loi \\
\hline \multirow{2}{*}{$\begin{array}{l}15.7 \\
15.3\end{array}$} & 106 & 55 & forme_gouverneme \\
\hline & 357 & 90 & espècé_horme \\
\hline \multirow{2}{*}{$\begin{array}{l}15.0 \\
15.0\end{array}$} & 65 & 43 & autorité_raison \\
\hline & 31 & 31 & attachement_coeu \\
\hline \multirow{2}{*}{$\begin{array}{l}14.8 \\
14.8\end{array}$} & 240 & 73 & coeur_objet \\
\hline & 228 & 71 & auteur_horme \\
\hline \multirow{2}{*}{$\begin{array}{l}14.6 \\
14.6\end{array}$} & 83 & 46 & autorité_loi \\
\hline & 42 & 35 & autorité_droit \\
\hline \multirow{2}{*}{$\begin{array}{l}14.4 \\
14.3\end{array}$} & 217 & & 230 coēur_horme \\
\hline & 108 & 50 & auteur_lettre \\
\hline \multirow{2}{*}{$\begin{array}{l}14.1 \\
14.1\end{array}$} & 72 & 42 & ferme_sexe \\
\hline & 37 & 32 & constitution_hom \\
\hline \multirow{2}{*}{$\begin{array}{l}14.0 \\
13.8\end{array}$} & 58 & 38 & autorité_hormēe \\
\hline & 27 & 27 & droit_souverain \\
\hline \multirow{2}{*}{$\begin{array}{l}13.7 \\
13.6\end{array}$} & 93 & 45 & citoyēn_homme \\
\hline & 426 & 88 & coeur_plaisir \\
\hline \multirow{2}{*}{$\begin{array}{l}13.6 \\
13.5\end{array}$} & 129 & 51 & chef_peuple \\
\hline & 26 & 26 & autrūi_mal \\
\hline \multirow{2}{*}{$\begin{array}{l}13.5 \\
13.4\end{array}$} & 112 & 48 & état_uñ \\
\hline & 804 & 121 & coeur_sentiment \\
\hline \multirow{2}{*}{$\begin{array}{l}13.3 \\
13.2\end{array}$} & 46 & 33 & citoyen_magistra \\
\hline & 44 & 32 & éducation_enfant \\
\hline \multirow{2}{*}{$\begin{array}{l}12.9 \\
12.9\end{array}$} & 72 & 38 & conseil_droit \\
\hline & 513 & 92 & coeur_räison \\
\hline 12.8 & 55 & 34 & expérience_horme \\
\hline
\end{tabular}

\begin{tabular}{|c|c|c|c|}
\hline \multirow{2}{*}{\multicolumn{4}{|c|}{ Cliquer sur un mot pour connatre sa spécificité dans }} \\
\hline & & & \\
\hline écart & corpu & 5 te & mot \\
\hline 27.8 & 490 & 171 & âme_flarme \\
\hline 23.7 & 82 & 72 & choix_roi \\
\hline 21.2 & 201 & 90 & amour haine \\
\hline 20.0 & 60 & 53 & conquềte tête \\
\hline 19.9 & 238 & 90 & gloire_victoire \\
\hline 19.7 & 2222 & 251 & amour_coeur \\
\hline 19.7 & 154 & 75 & envie_vie \\
\hline 19.4 & 93 & 61 & coeur_rigueur \\
\hline 19.2 & 1302 & 185 & arnour-jour \\
\hline 18.6 & 213 & 80 & coeur_flamme \\
\hline 18.5 & 137 & 67 & coeur_vainqueur \\
\hline 18.3 & 306 & 91 & coeur_roi \\
\hline 17.6 & 73 & 50 & crime_victime \\
\hline 17.4 & 41 & 39 & haine_reine \\
\hline 17.2 & 126 & 60 & choix_coeur \\
\hline 17.1 & 178 & 68 & coeur_seigneur \\
\hline 17.1 & 129 & 60 & coeur_empire \\
\hline 16.9 & 73 & 48 & amour_choix \\
\hline 16.9 & 220 & 73 & amour_roi \\
\hline 16.8 & 97 & 53 & amour_ardeur \\
\hline 16.3 & 82 & 48 & haine peine \\
\hline 16.3 & 118 & 55 & coeur_haine \\
\hline 16.2 & 70 & 45 & choix \\
\hline 16.1 & 38 & 35 & attentat_etat \\
\hline 16.0 & 106 & 52 & effort_mort \\
\hline 15.9 & 103 & 51 & amour_faveur \\
\hline 15.8 & 236 & 70 & coeur_gloire \\
\hline 15.6 & 139 & 56 & coeur_voeu \\
\hline 15.4 & 97 & 48 & amour_voeu \\
\hline 15.4 & 188 & 62 & douleur_malheur \\
\hline 15.3 & 57 & 39 & époux_māin \\
\hline 15.0 & 104 & 48 & amour_espoir \\
\hline 14.9 & 40 & 33 & couronne personn \\
\hline 14.9 & 40 & 33 & coeur_tyran \\
\hline 14.9 & 174 & 58 & âme_rōi \\
\hline 14.8 & 77 & 42 & amour_hymen \\
\hline 14.8 & 155 & 55 & amour_crime \\
\hline
\end{tabular}

On n'ignore pas que dans ces changements, dont témoignent les cooccurrences, le genre, lui-même soumis aux variations du temps et de la mode, a une large influence qui s'impose à l'écrivain. C'est pourtant l'écrivain qui choisit son genre ou qui l'invente, suivant ses goûts et ses dons. Et les spécificités de chacun apparaissent plus clairement dans les couples que dans les mots isolés. Ainsi dans le tableau 6, qui met en parallèle Corneille et Rousseau, on trouverait sans difficulté des mots appartenant aux deux listes, comme cœur par exemple. Mais chez Corneille le cæur a ses raisons et ses cooccurrents qui ne sont pas les mêmes chez Rousseau. On chercherait en vain un couple qui soit identique chez les deux 
écrivains. Les éléments peuvent être communs aux deux, non les combinaisons. Bien entendu si l'on avait rapproché Corneille de Racine, les rencontres seraient plus nombreuses, notamment celles que la rime engendre: âme-flamme, choix-roi, conquêtetête, gloire-victoire (ce sont les quatre premiers couples chez Corneille), gloire-victoire, prince-province, crime-victime, gloiremémoire, cœur-vainqueur, alarme-larme (ces couples sont en tête des spécificités de Racine).

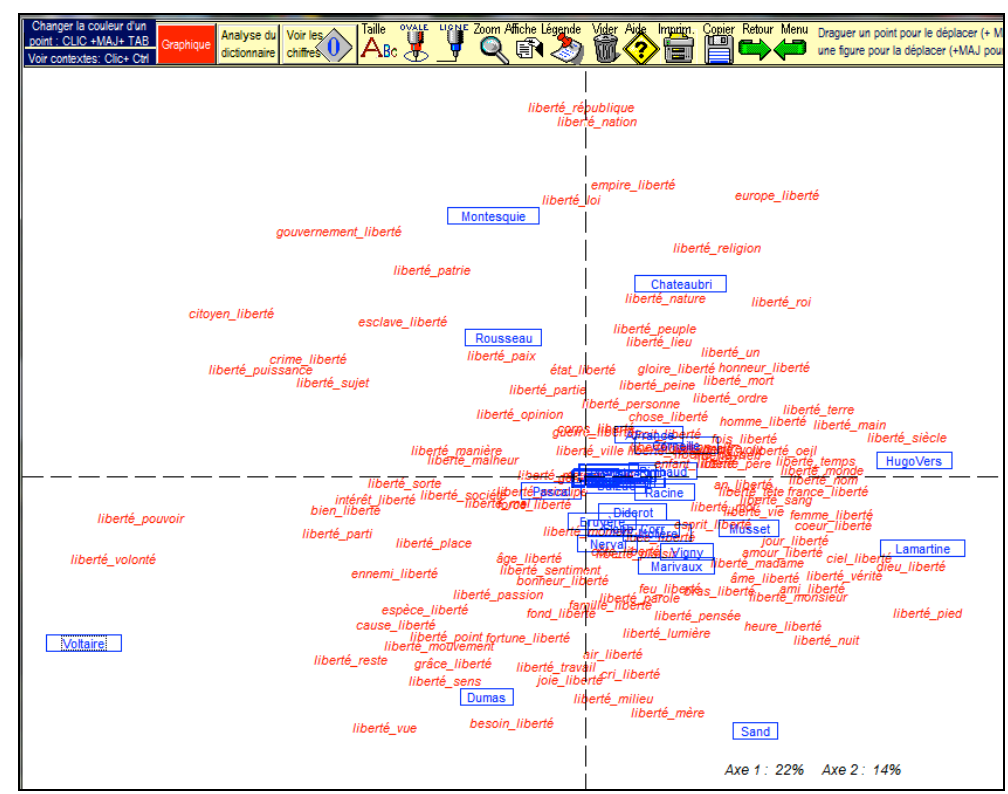

Figure 18. Analyse factorielle des couples où règne la liberté

Parmi les outils disponibles l'analyse factorielle reste la pièce maîtresse. Elle peut ici s'appliquer à la phraséologie qui tourne autour d'un mot. On s'est longtemps battu sur le crédit incertain qu'on pouvait donner au lemme, en invoquant les nuances de sens qui s'attachent au singulier et au pluriel (peuple versus peuples, liberté versus libertés). Lemme ou simple graphie, un mot isolé n'a guère de substance. Il vit de son environnement. Or la cooccurrence, en lui restituant une part de son entourage, lui redonne sens et signification. Ainsi le débat sur liberté et libertés s'efface quand mille libertés différentes jaillissent du texte en demandant classement et typologie. La 


\section{É. BRUNET}

figure 18 éclaire le visage multiple où s'incarne le mot au fil des siècles. Les écrivains antérieurs aux Lumières ne lui donnent pas grand crédit et ils s'agglutinent au centre du graphique sans prendre parti. C'est Montesquieu au haut du graphique qui donne au mot ses lettres de noblesse, en l'associant au vocabulaire politique (république, nation, empire, loi, gouvernement). A sa gauche Rousseau oriente le terme dans un sens plus social et plus affectif (patrie, paix, esclave, crime, opinion), tandis qu'à droite Chateaubriand rapproche la liberté des questions religieuses et monarchiques (religion, roi, peuple, Europe). Dans la moitié basse de la figure, l'opposition est encore celle de la chronologie : Voltaire à gauche et Lamartine à droite, avec l'appui de quelques écrivains engagés comme Hugo et Sand. Chez eux la liberté est moins une notion théorique de l'économie politique qu'un étendard sous lequel il convient de se battre, à l'image du tableau célèbre La liberté guidant le peuple. Le combat de Voltaire (qui n'a pas oublié son séjour à La Bastille) est celui de la volonté, contre l'ennemi, contre le pouvoir, contre le parti. Celui de Lamartine, de Sand et de Hugo, est tout aussi sincère, mais il s'entoure de notions ou sentiments plus vagues où la liberté est un ingrédient qu'on utilise dans toutes les sauces et qui peut s'associer à l'amour, à la femme, à Dieu, à la vérité, etc. Le cas de Dumas en position centrale est un peu particulier : la liberté y est attachée trop rudement à la prison. C'est un élément d'une intrigue qui multiplie les emprisonnements, les évasions et les délivrances.

En conclusion il reste à prolonger l'expérimentation des méthodes fondées sur la cooccurrence. Les segments répétés du logiciel Lexico ont ouvert une brèche, et le succès d'Alceste a montré qu'il y avait une attente de la communauté scientifique, que voudrait satisfaire, dans une modeste mesure et parmi beaucoup d'autres, notre contribution. A cet égard, à l'heure où nous mettons sous presse, le logiciel Hyperbase, au-delà des nouvelles fonctionnalités présentées ici, permet de s'articuler par simple clic avec le logiciel open-source Gephi afin de proposer une meilleure visualisation des données relationnelles complexes que sont, par essence, le treillis cooccurrentiel d'un texte. 